\title{
Measurement of Electron Return Effect and Skin Dose Reduction by a Bolus in an Anthropomorphic Physical Phantom under a Magnetic Resonance Guided Linear Accelerator (MR-LINAC) System
}

\author{
Eun Young Han1, Zhifei Wen¹, Hannah J. Lee1, Arnold dela Cruz Paulino1, Choonsik Lee² \\ ${ }^{1}$ Department of Radiation Physics, The University of Texas MD Anderson Cancer Center, Houston, TX, USA \\ ${ }^{2}$ Division of Cancer Epidemiology and Genetics, National Cancer Institute, National Institutes of Health, Rockville, USA \\ Email: ehan@mdanderson.org, Zwen@mdanderson.org,HJLee1@mdanderson.org, APaulino@mdanderson.org, \\ Leechoonsik@mail.nih.gov
}

How to cite this paper: Han, E.Y., Wen, Z.F., Lee, H.J., dela Cruz Paulino, A. and Lee, C. (2018) Measurement of Electron Return Effect and Skin Dose Reduction by a Bolus in an Anthropomorphic Physical Phantom under a Magnetic Resonance Guided Linear Accelerator (MR-LINAC) System. International Journal of Medical Physics, Clinical Engineering and Radiation Oncology, 7, 339-346.

https://doi.org/10.4236/ijmpcero.2018.73028

Received: July 6, 2018

Accepted: August 11, 2018

Published: August 14, 2018

Copyright ( 2018 by authors and Scientific Research Publishing Inc. This work is licensed under the Creative Commons Attribution International License (CC BY 4.0).

http://creativecommons.org/licenses/by/4.0/

\begin{abstract}
Background: Magnetic resonance image-guided radiation therapy (MRIGRT) promises more precise and effective radiation treatments compared to conventional IGRT by using real-time on-board MR imaging. Under the influence of a magnetic field, however, secondary electrons exiting a surface can be forced in a circular path and re-enter the medium, resulting in dose increase at a beam-exit surface, called the electron return effect (ERE). The purpose of the study is to compare the exit skin dose computed by Monte Carlo dose calculation with measurements using an adult anthropomorphic phantom and to measure the effect of skin dose reduction by adding 1 $\mathrm{cm}$-thick bolus. Method: The plan was compared with measurements using an adult anthropomorphic phantom combined with radiochromic films and thermoluminescent dosimeters. We also measured the skin dose reduction by adding $1 \mathrm{~cm}$-thick bolus on the frontal surface of the phantom. Results: We found that $1 \mathrm{~cm}$-thick bolus reduced the skin dose by up to $20 \%$ both in measurements and calculations. The plan was found to overestimate the measured skin dose by about $10 \%$ and there was no significant difference in the bolus effect between the breast skin and the skin (without breast attachment) doses. Conclusion: In conclusion, we confirmed the ERE effect on the anthropomorphic phantom under the magnetic field and the exit skin dose reduction by adding a bolus. Skin dose measurements using anthropomorphic phantom may be helpful to evaluate more realistic skin dose and the bo-
\end{abstract}


lus effect in the magnetic field.

\section{Keywords}

MR-Linac, Electron Return Effect, Skin Dose

\section{Background}

Magnetic resonance image-guided radiation therapy (MR-IGRT) is designed to provide more precise and effective radiation treatments compared to conventional computed tomography (CT)- or cone beam CT-based IGRT by using real-time on-board MR imaging [1] [2] [3] [4]. However, it is reported that under the influence of a magnetic field, secondary electrons exiting a surface can be directed in a circular path and re-entering the medium, resulting in dose increase at a beam-exit surface, called the electron return effect (ERE) [1] [2] [3] [4]. Several authors confirmed the ERE by using Monte Carlo (MC) simulation as well as measurements. Raaijmakers et al. [2] [3] demonstrated the ERE by MC simulation, which was also validated by film measurement in sold water phantoms. Chen et al. investigated the dosimetric effect of ERE at air-tissue and lung-tissue interfaces during intensity modulated radiation therapy (IMRT) [5]. Woodings et al. conducted a comprehensive dosimetric characterization of the Elekta 1.5T MR-Linac beam for clinical application [6]. To address the unintended skin dose increase by ERE, Oborn et al. [7] suggested the use of 1 $\mathrm{cm}$-thick bolus significantly mitigates the exit dose increase. It is now important to measure the skin dose increase by the ERE and its reduction by a bolus using physical anthropomorphic phantoms, in more realistic radiotherapy condition.

The Ekelta MR-Linac (Elekta, Stockholm, Sweden) combines an Elekta Linac and a 1.5 T MR scanner (Philips Healthcare, Best, Netherlands) [8]. Within the system, a 7-MV radiation beam is oriented perpendicular to the magnetic field of the MR scanner. The rotation axis of the treatment beam gantry, which is the same as the patient's craniocaudal axis on the treatment couch, is parallel to the magnetic field. The purpose of the current study is to measure the exit skin dose using an adult female physical anthropomorphic phantom with thermoluminescent dosimeter (TLD) and radiochromic film for antero-posterior (AP) and postero-anterior (PA) treatment beams using the MR-Linac machine. We then investigated the skin dose reduction by adding $1 \mathrm{~cm}$-thick bolus on the frontal surface of the phantoms irradiated by the PA beam. The measured doses were also compared with a Monte Carlo (MC) dose calculation module built in the MR-Linac system.

\section{Materials and Methods}

\subsection{Skin Dose Measurement with Phantom}

We employed an adult female anthropomorphic Alderson RANDO phantom 
(The Phantom Laboratory, Salem, New York, NY) for measurements of the skin dose. We removed two breast attachments to investigate the impact without the breast tissue on skin dose and we called it as "adult male phantom" for the sake of simple notation in this study. We also adopted a bolus of $1 \mathrm{~cm}$ thickness for both adult male and female phantoms (Figure 1). The skin dose was measured using TLD (TLD-100; Thermo Fisher Scientific, Waltham, MA) and gafchromic film (EBT3; Ashland, Bridgewater, NJ) for four phantom setups.

For the adult male phantom (Figure 1(a) and Figure 1(b)), three locations were selected for the TLD and film measurements: the Central Axis (CAX), $5 \mathrm{~cm}$ to the right of the CAX, and $6 \mathrm{~cm}$ to the left of the CAX. We had to move the right position by $1 \mathrm{~cm}$ left to avoid the possible air gap caused by the engraved letters on the phantom. A square array of four individually sealed TLD powder pouches with total dimension of $25 \mathrm{~mm} \times 25 \mathrm{~mm} \times 0.67 \mathrm{~mm}$ was placed at the three locations. The thickness of the pouches $(0.67 \mathrm{~mm} \pm 0.085 \mathrm{~mm})$ was measured with a digital caliper for 35 pouch samples. The EBT3 gafchromic film was cut in the size of $25 \mathrm{~mm} \times 25 \mathrm{~mm} \times 0.23 \mathrm{~mm}$. We adopted a tissue-equivalent bolus (CIVCO Medical Solutions, Coralville, IA) with density of $1.02-1.03$

(a) Adult male phantom without bolus

(b) Adult male phantom with bolus

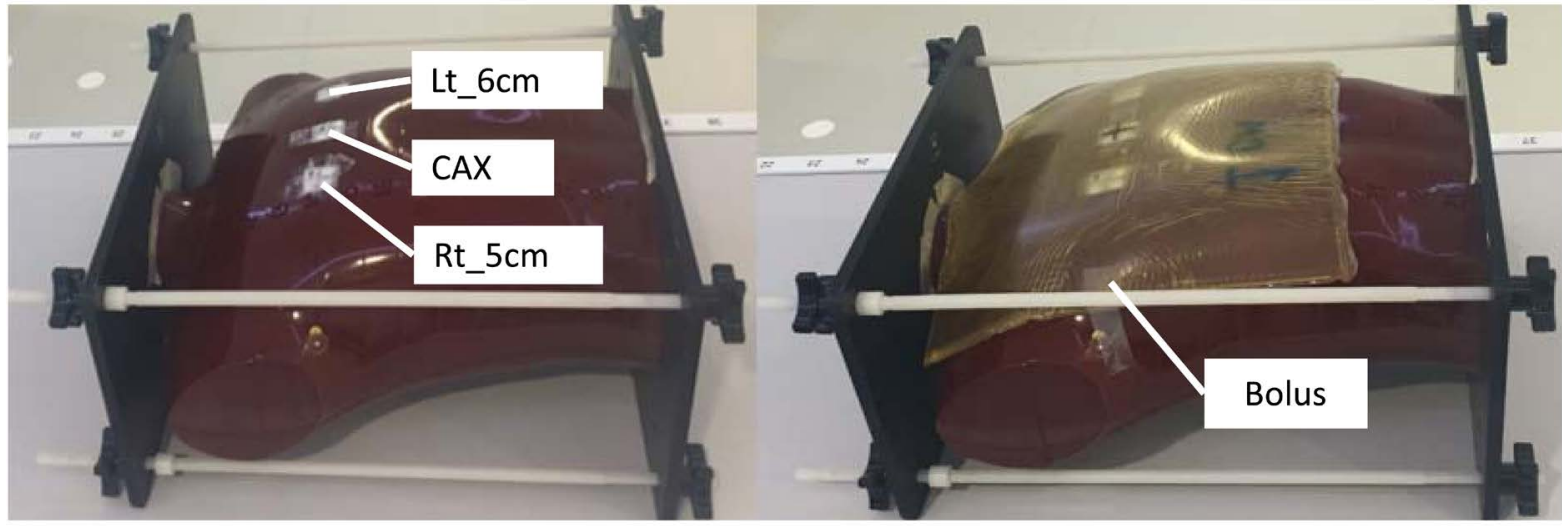

(c) Adult female phantom without bolus

(d) Adult female phantom with bolus

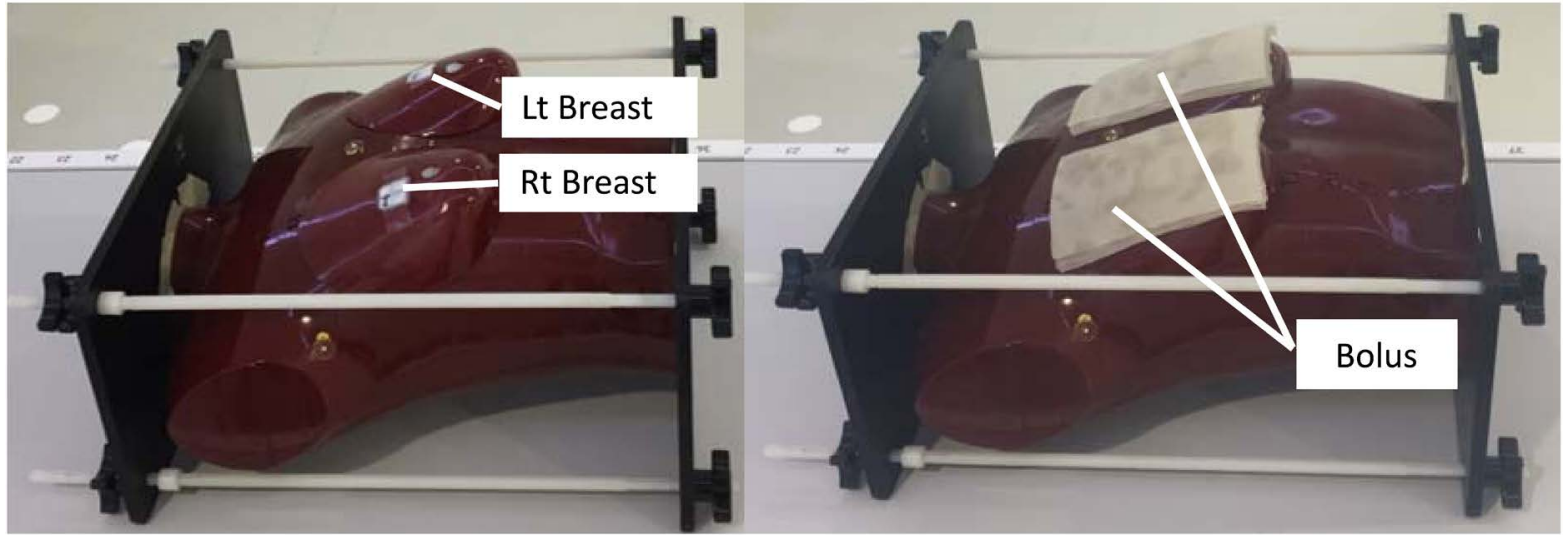

Figure 1. The adult male phantom (a) with and (b) without $1 \mathrm{~cm}$-thick bolus and the adult female phantom with breast attachment (c) with and (d) without $1 \mathrm{~cm}$-thick bolus. Three TLDs (left, center, and right) were attached to the adult male phantom and two TLDs (left and right) were used for the adult female phantom. 
$\mathrm{g} / \mathrm{cm}^{3}$. Two sets of measurements were obtained: the first measurement for AP and PA beams without bolus (Figure 1(a)) and the second measurement for AP beam without bolus and PA beam with bolus on the frontal surface (Figure 1(b)). Each measurement was performed twice.

For the adult female phantom with the breast attachment, two measurement positions were selected as shown in Figure 1(c) and Figure 1(d): right breast (1 $\mathrm{cm}$ superior and $1.5 \mathrm{~cm}$ right to the center of the right plastic screw) and left breast ( $1 \mathrm{~cm}$ superior and $1.5 \mathrm{~cm}$ left to the center of the left plastic screw). We selected a relatively flat surface to facilitate the placement of bolus and the attachment of TLDs. To avoid air gap between the bolus and still curved surface of the breast attachment, more elastic bolus (Superflab; Mick Radio-Nuclear Instruments, Mount Vernon, NY) was used with a density $\left(1.02-1.03 \mathrm{~g} / \mathrm{cm}^{3}\right)$. The original sheet with the size of $30 \mathrm{~cm} \times 30 \mathrm{~cm} \times 0.3 \mathrm{~cm}$ was cut into six equal parts $(10 \mathrm{~cm} \times 15 \mathrm{~cm}$ each); three sheets were stacked up to $9 \mathrm{~mm}$ thickness for each breast (Figure $1(\mathrm{~d})$ ).

The TLDs and films were calibrated using solid water phantoms in the MR-Linac on the same day. The calibration was performed by measuring dose with a $7 \mathrm{MV}$ beam with the field size of $10 \times 10 \mathrm{~cm}^{2}$ at $10 \mathrm{~cm}$ depth with a source to detector distance of $143.5 \mathrm{~cm}$. Difference doses, 0, 20, 50, 100, 200, and $500 \mathrm{cGy}$, were delivered to the water phantom. Three individually sealed TLD pouches were positioned on the CAX and irradiated at the same time. A calibration curve from average TLD readings versus delivered dose was generated. Using the EBT3 film, the dose was measured twice for the film with the size of $2 \times 2 \mathrm{~cm}^{2}$ at the same calibration geometry with the TLD. The films were scanned using a 10,000 XL flatbed scanner (Epson, Long Beach, CA), and a film calibration file was generated using the FilmQA Pro software program (Ashland, Bridgewater, NJ).

We planned AP-PA beam alignment with relatively large field size, 24 (axial direction) $\times 22$ (craniocaudal direction) $\mathrm{cm}^{2}$, for the skin dose measurements, which are reported to maximize ERE [4]. The illustrative treatment plans were generated for the four phantom setups (Figure 1) using unblocked thoracic fields of $7 \mathrm{MV}$ beam in a $1.5 \mathrm{~T}$ magnetic field. The bolus was simulated as water in the plans. The prescribed dose was $3000 \mathrm{cGy}$ in 10 fractions. The dose was normalized at the midline of the upper torso in the phantom. Planned monitor unit (MU) were $126 \mathrm{MU}(\mathrm{AP})$ and $163 \mathrm{MU}$ (PA) for the adult male phantom and $114 \mathrm{MU}$ (AP) and $203 \mathrm{MU}$ (PA) for the adult female phantom with breast attachments. The MU settings were identical for the plans with and without bolus. Higher MU for PA beam were used because the location of beam CAX was fixed at $13 \mathrm{~cm}$ above the couch top within the phantom of $18 \mathrm{~cm}$ thickness while both beams are equally weighted.

\subsection{Skin dose Calculation with Monaco TPS}

We compared the measured skin doses with those calculated from the MC dose calculation module, built in the MR-Linac system: the Monaco treatment plan- 
ning system (research version 5.19.02). The MC module is based on a Graphical Processing Unit Monte Carlo Dose calculation (GPUMCD) algorithm [9] [10]. This new dose calculation algorithm was benchmarked with other MC dose calculation codes and measurements by other authors [9] [10] [11]. The adult male and female phantoms were scanned with $2 \mathrm{~mm}$ slice thickness with a resolution of $1.07 \mathrm{~mm}$ using a Brilliance $16 \mathrm{CT}$ scanner (Philips Healthcare, Amsterdam, Netherlands) and imported into the Monaco TPS for the skin dose calculations.

The International Commission on Radiological Protection (ICRP) reports that the basal cells of the epidermis are at radiogenic risk, which is within a range from 50 to $100 \mu \mathrm{m}$ and recommends the use of $70 \mu \mathrm{m}$ as a reasonable mean depth of the radiosensitive cell layer [12] [13]. However, it was impossible to define the thin layer in our calculation due to the limited resolution of dose grid. Therefore we had to define more practical region of interest in calculations, which was supposed to be identical to the measurement conditions described before. Considering expectedly sharp dose gradient close to the skin-air interface, we decided to define $0.5 \mathrm{~mm}$ above and $0.5 \mathrm{~mm}$ below the phantom surface as a computational surrogate for the actual skin thickness. The dose grid of $1 \times 1$ $\times 1 \mathrm{~mm}^{3}$ was used to accurately calculate the skin dose with statistical uncertainty of $\leq 2 \%$.

\section{Results and Discussion}

Table 1 shows the comparison of the calculated mean anterior skin dose under no magnetic field (0T), 1.5 T magnetic field and $1.5 \mathrm{~T}$ magnetic field with $1-\mathrm{cm}$ bolus on the frontal skin in the adult male and female phantom. We found that there is $\sim 34 \%$ of skin dose increase under $1.5 \mathrm{~T}$ and this is consistent with other authors' findings [2] [3] [4] [11] [14]. Also, we found that there is $~ 20 \%$ skin dose reduction by adding a $1-\mathrm{cm}$ bolus on the exit skin.

Figure 2 shows the skin dose comparison between the measurements and the plans for the RANDO phantom without the breast attachment. Even though the effective measurement depths are different due to the difference of TLD and film thickness, we found that the measurements from film and TLD agree within about $5 \%$, which was within the measurement uncertainty as shown in the error bars in Figure 2. The possible reason for this is that the measured values of both detectors were averaged over the detector area of $25 \mathrm{~mm} \times 25 \mathrm{~mm}$.

In Figure 2(a), the right and left skin doses were 7\% - 14\% higher than the dose at the CAX and this was caused by lower attenuation of the PA beam by the

Table 1. Calculated mean anterior skin dose under no magnetic field, 1.5T magnetic field and $1.5 \mathrm{~T}$ magnetic field with $1-\mathrm{cm}$ bolus on the frontal skin in the adult male and female phantom.

\begin{tabular}{cccc}
\hline & OT & $1.5 \mathrm{~T}$ & $1.5 \mathrm{~T}$ with $1 \mathrm{~cm}$ bolus \\
\hline Adult male mean skin dose (cGy) & 154.3 & 207.2 & 176.1 \\
Adult female mean skin dose (cGy) & 147.3 & 197.8 & 177.6 \\
\hline
\end{tabular}


(a) Adult male phantom

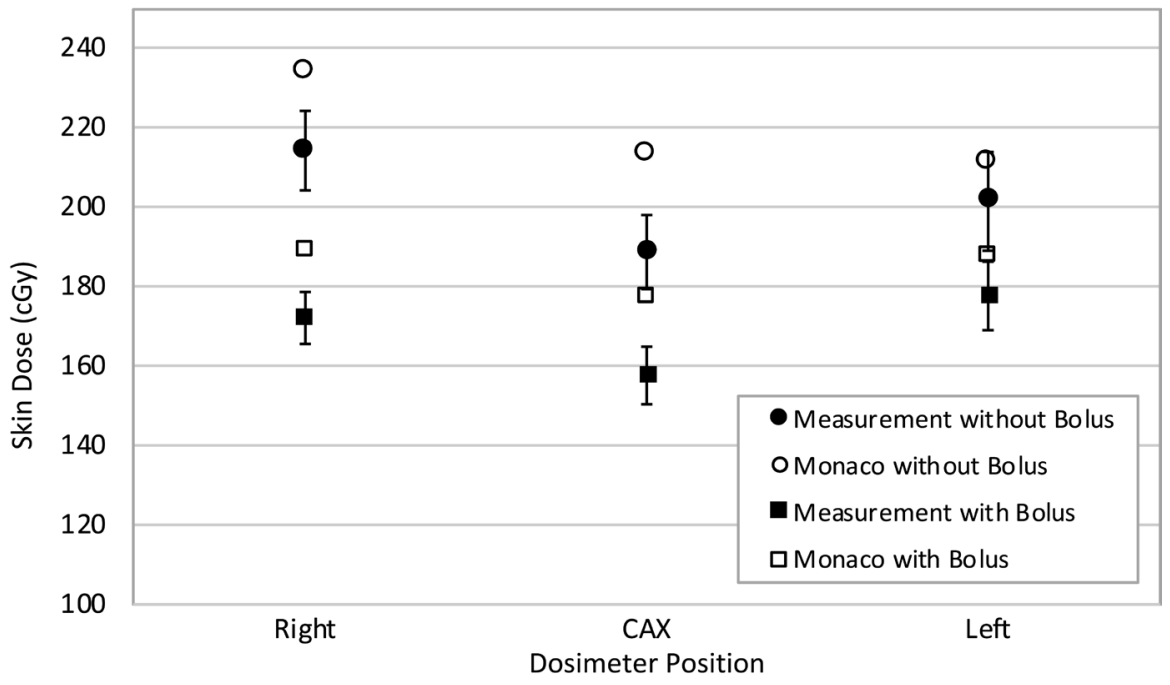

(b) Adult female phantom

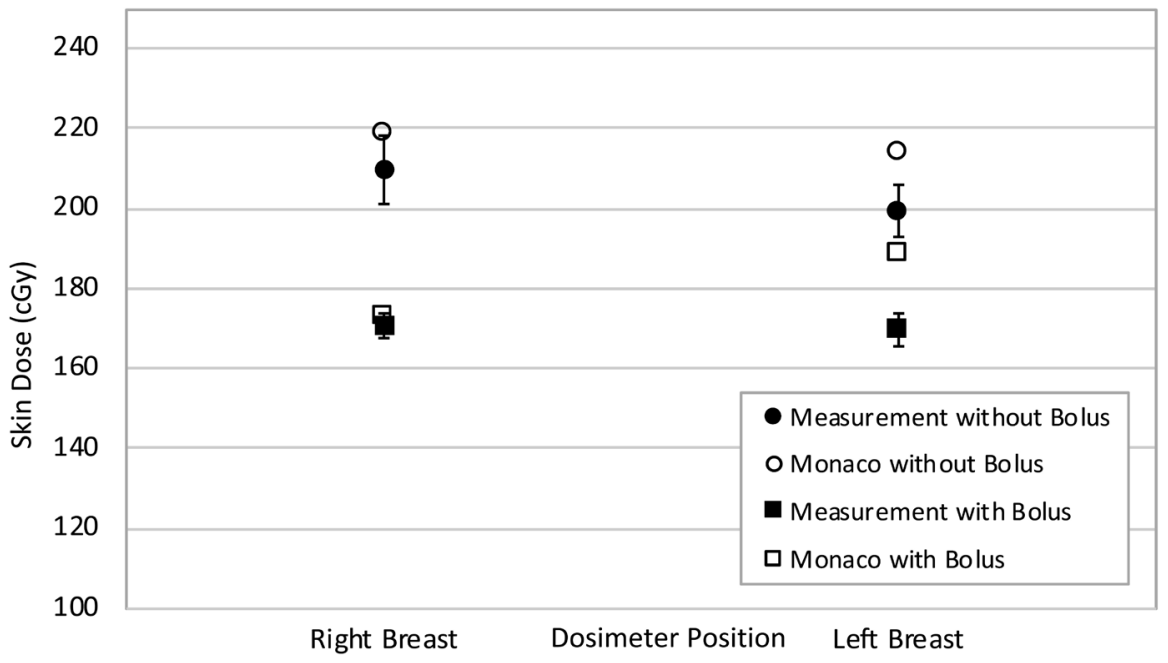

Figure 2. Comparison of the skin doses in (a) the adult male phantom at the three locations (right, CAX, and left) and in (b) adult female phantom with breast attachment at the two locations (right and left) between measurements and Monaco calculations with and without bolus.

lungs comparing to where the CAX is located. Also, the right measured and calculated skin doses were slightly higher than the left skin dose and this is caused by electrons moving in circular path before returning to the phantom under the magnetic field [2] [4].

The $1 \mathrm{~cm}$-thick bolus reduced the skin dose measurements by $20 \%, 16 \%$, and $12 \%$ and the calculated skin doses by $19 \%, 17 \%$, and $11 \%$ at right, CAX, and left positions, respectively. The calculated skin dose overall overestimated the measurements by about $10 \%$, however it must be noted that the estimation of calculated skin dose could be driven by the statistical uncertainty of the MC calculation and the protocol of skin contouring because of the sharp dose gradient close to the skin-air interface and the limited dose grid size. 
Figure 2(b) shows the breast skin dose comparison between measurements and Monaco calculations with and without bolus for the phantom with the breast attachment. The $0.9 \mathrm{~cm}$-thick bolus reduced the measured skin dose by $19 \%$ (right) and $15 \%$ (left) and the calculated skin dose by $21 \%$ (right) and $12 \%$ (left), which are about the similar reduction rate with the case of the adult male phantom.

It should be noted that the skin dose increase by ERE and dose reduction by a bolus may vary depending on the size of the adult patient.

\section{Conclusion}

We confirmed the ERE effect on the anthropomorphic phantom under the magnetic field and found that the suggested skin dose estimation method overall overestimated the skin dose by about $10 \%$ comparing to the measurements. We also confirmed that $1 \mathrm{~cm}$-thick bolus could reduce the skin dose by up to $20 \%$ both in measurements and calculations. Skin dose measurements using anthropomorphic phantom may be helpful to evaluate more realistic skin dose and the bolus effect in the magnetic field.

\section{Acknowledgements}

This project used a research version of Monaco TPS provided by Elekta ${ }^{\mathrm{TM}}$.

\section{Conflicts of Interest}

The authors declare no conflicts of interest regarding the publication of this paper.

\section{References}

[1] Raaijmakers, A.J., et al. (2007) Dose Optimization for the MRI-Accelerator: IMRT in the Presence of a Magnetic Field. Physics in Medicine \& Biology, 52, 7045-7054. https://doi.org/10.1088/0031-9155/52/23/018

[2] Raaijmakers, A.J., Raaymakers, B.W. and Lagendijk, J.J. (2005) Integrating a MRI Scanner with a 6 MV Radiotherapy Accelerator: Dose Increase at Tissue-Air Interfaces in a Lateral Magnetic Field Due to Returning Electrons. Physics in Medicine \& Biology, 50, 1363-1376. https://doi.org/10.1088/0031-9155/50/7/002

[3] Raaijmakers, A.J., Raaymakers, B.W. and Lagendijk, J.J. (2007) Experimental Verification of Magnetic Field Dose Effects for the MRI-Accelerator. Physics in Medicine \& Biology, 52, 4283-4291. https://doi.org/10.1088/0031-9155/52/14/017

[4] Raaijmakers, A.J., Raaymakers, B.W. and Lagendijk, J.J. (2008) Magnetic-Field-Induced Dose Effects in MR-Guided Radiotherapy Systems: Dependence on the Magnetic Field Strength. Physics in Medicine \& Biology, 53, 909-923. https://doi.org/10.1088/0031-9155/53/4/006

[5] Chen, X.F., et al. (2016) Technical Note: Dose Effects of 1.5 T Transverse Magnetic Field on Tissue Interfaces in MRI-Guided Radiotherapy. Medical Physics, 43, 4797-4802. https://doi.org/10.1118/1.4959534

[6] Woodings, S.J., et al. (2018) Beam Characterisation of the 1.5 T MRI-Linac. Physics in Medicine \& Biology, 63, 085015. https://doi.org/10.1088/1361-6560/aab566 
[7] Oborn, B.M., et al. (2010) Monte Carlo Characterization of Skin Doses in 6 MV Transverse Field MRI-Linac Systems: Effect of Field Size, Surface Orientation, Magnetic Field Strength, and Exit Bolus. Medical Physics, 37, 5208-5217. https://doi.org/10.1118/1.3488980

[8] Raaymakers, B.W., et al. (2004) Integrating a MRI Scanner with a 6 MV Radiotherapy Accelerator: Dose Deposition in a Transverse Magnetic Field. Physics in Medicine \& Biology, 49, 4109-4118. https://doi.org/10.1088/0031-9155/49/17/019

[9] Hissoiny, S., et al. (2011) GPUMCD: A New GPU-Oriented Monte Carlo Dose Calculation Platform. Medical Physics, 38, 754-764. https://doi.org/10.1118/1.3539725

[10] Hissoiny, S., et al. (2011) Fast Dose Calculation in Magnetic Fields with GPUMCD. Physics in Medicine \& Biology, 56, 5119-5129. https://doi.org/10.1088/0031-9155/56/16/003

[11] Ahmad, S.B., et al. (2016) Evaluation of a Commercial MRI Linac Based Monte Carlo Dose Calculation Algorithm with GEANT4. Medical Physics, 43, 894-907. https://doi.org/10.1118/1.4939808

[12] ICRP (1998) Radiation Dose to Patients from Radiopharmaceuticals (Addendum 2 to ICRP Publication 53). Annals of the ICRP, 28, 1-126.

[13] Petoussi-Henss, N., et al. (2010) ICRP Publication 116. Conversion Coefficients for Radiological Protection Quantities for External Radiation Exposures. Annals of the ICRP, 40, 1-257. https://doi.org/10.1016/j.icrp.2011.10.001

[14] Raaijmakers, A.J., et al. (2007) Integrating a MRI Scanner with a 6 MV Radiotherapy Accelerator: Impact of the Surface Orientation on the Entrance and Exit Dose Due to the Transverse Magnetic Field. Physics in Medicine \& Biology, 52, 929-939. https://doi.org/10.1088/0031-9155/52/4/005 